\title{
ERRATUM
}

Xia Wang • Ting Jiang • Jinpeng Yuan •

Chuange Cheng $\cdot$ Jianhua Liu

Junbo Shi $\cdot$ Rusong Zhao

\section{Determination of volatile residual solvents in pharmaceutical products by headspace liquid-phase microextraction gas chromatography-flame ionization detector}

Published online: 10 August 2006

(C) Springer-Verlag 2006

Please regard the following corrections in this article:

Page 1, "Abstract", line 7:

$0.2-2.0 \mathrm{mg} \mathrm{l}^{-1}$ should be $0.2-6.0 \mathrm{mg} \mathrm{l}^{-1}$.

Page 4, section "Linear range, limits of detection and repeatability"; line 6:

0.2 to $2.0 \mathrm{mg}^{-1}$ should be 0.2 to $6.0 \mathrm{mg} \mathrm{l}^{-1}$.

Page 4, new version of Table 2.

Table 2 Linear range, correlation coefficient $\left(R^{2}\right)$, precision, and limits of detection (LODs) using HS-LPME-GC-FID analysis

\begin{tabular}{lllllll}
\hline Solvent & Linear range $\left(\mathrm{mg} \mathrm{l}^{-1}\right)$ & $\mathrm{R}^{2}$ & $\mathrm{RSD}(\%)(n=5)$ & $\mathrm{LOD}\left(\mathrm{mg} \mathrm{l}^{-1}\right)$ & $\left.\mathrm{LOD}^{\mathrm{a}}(\mu \mathrm{g} \mathrm{g})^{-1}\right)$ & $\mathrm{SPME} \mathrm{LOD}^{\mathrm{b}}\left(\mathrm{mg} \mathrm{l}^{-1}\right)$ \\
\hline Tetrahydrofuran & $1-100$ & 0.9975 & 9.4 & 0.2 & 2.0 & 0.03 \\
Methanol & $10-1000$ & 0.9986 & 12.1 & 6.0 & 60 & - \\
Dichloromethane & $1-100$ & 0.9890 & 6.8 & 0.2 & 2.0 & 0.005 \\
Ethanol & $5-500$ & 0.9978 & 7.0 & 3.0 & 30 & 0.5 \\
\hline
\end{tabular}

${ }^{\mathrm{a}} \mathrm{LOD}$ in pharmaceutical product $(0.5-\mathrm{g}$ sample)

${ }^{\mathrm{b}}$ Data from [4]

The online version of the original article can be found at http://dx. doi.org.10.1007/s00216-005-0506-2.

X. Wang $(\bowtie) \cdot$ T. Jiang $\cdot$ J. Yuan ·

C. Cheng $\cdot$ J. Liu $\cdot$ J. Shi $\cdot$ R. Zhao

Key Laboratory for Applied Technology of Sophisticated

Analytical Instruments of Shandong Province,

Analysis and Test Center, Shandong Academy of Sciences,

Jinan, Shandong, 250014, People's Republic of China

e-mail: xia-w21cn0@163.com 